\title{
On the validity of variational inequalities for obstacle problems with non-standard growth
}

\author{
Michela Eleuteri and Antonia Passarelli di Napoli
}

\begin{abstract}
The aim of the paper is to show that the solutions to variational problems with non-standard growth conditions satisfy a corresponding variational inequality expressed in terms of a duality formula between the constrained minimizers and the corresponding dual maximizers, without any smallness assumptions on the gap between growth and coercitivity exponents. Our results rely on techniques based on Convex Analysis that consist in establishing pointwise relations that are preserved passing to the limit. We point out that we are able to deal with very general obstacle quasi-continuous up to a subset of zero capacity.
\end{abstract}

\section{Epästandardin kasvun esteongelmia koskevien variaatioepäyhtälöiden voimassaolosta}

Tiivistelmä. Tutkimuksen tavoitteena on osoittaa, että epästandardeja kasvuehtoja sisältävien variaatio-ongelmien ratkaisut toteuttavat vastaavan variaatioepäyhtälön, joka muotoillaan duaalisuuskaavana rajoitetusehdon toteuttavien minimoijien ja vastaavien duaalimaksimoijien välillä. Tämä pätee ilman mitään kasvu- ja koersiivisuuseksponenttien välimatkaa koskevia pienuusoletuksia. Tuloksemme nojaavat konveksiin analyysiin perustuviin tekniikoihin, joissa kyse on rajankäynnissä säilyvien pisteittäisten suhteiden osoittamisesta. On huomionarvoista, että pystymme käsittelemään hyvin yleisiä esteitä, jotka ovat nollakapasiteettista osajoukkoa vaille kvasijatkuvia.

\section{Introduction}

More than 30 years ago, the celebrated papers by Marcellini [17, 18] opened the way to the study of the regularity properties of minimizers of integral functionals with non-standard growth conditions. Since then, many contributions appeared in several directions and many problems have been solved; however not all the questions have been addressed in an exhaustive way, in particular for what concerns the obstacle problems.

One of these problems deals with the relation between minima and extremals, as long as it is well known that, for both the constrained and unconstrained setting, the regularity of the solutions often comes from the fact that are also extremals, i.e. they solve a corresponding variational inequality or equality. While in the case of standard growth conditions the situation is well established (see for instance [8]), in the case of non-standard growth conditions, already for unconstrained minimizers the relation between extremals and minima is an issue that requires a careful investigation.

Some years ago Carozza, Kristensen and Passarelli di Napoli investigated such a topic in the case of minimizers of convex integral functionals, with the aim of showing that these minimizers are characterized to be the energy solutions to the Euler-Lagrange system for the functionals under non-standard growth conditions

https://doi.org/10.54330/afm.114655

2020 Mathematics Subject Classification: Primary 35J87, 49J40, 47J20.

Key words: Variational inequalities, obstacle problems, duality formulas.

(c) 2022 The Finnish Mathematical Society 
[2]. The main tool there has been a suitable regularization procedure: the integrand $F$ has been approximated by a sequence of strictly convex and uniformly elliptic integrands $F_{k}$, satisfying standard $p$-growth conditions, whose minimizers $u_{k}$ strongly converge to the minimizer $u$ in $W^{1, p}$; then, to every such minimizer $u_{k}$, according to the standard duality theory for convex problems, it has been associated a row-wise solenoidal matrix field denoted by $\sigma_{k}$. Finally, for the pairing $\left(D u_{k}, \sigma_{k}\right)$, suitable pointwise estimates that are preserved in passing to the limit have been proved. Such estimates then provide conditions in order for the Euler-Lagrange system to hold for an $F$-minimizer. In a subsequent paper [3] the same achievement has been carried on under more general growth assumptions, by means of the use of Ekeland variational principle and Young measures, to obtain the necessary estimates for the pairing $\left(D u_{k}, \sigma_{k}\right)$ to be able to pass to the limit.

In this paper we address the analogue issue in the case of constrained minimizers since, for the so called obstacle problem, the situation has not been well established yet. In the recent paper [5] the authors, dealing with the question of Lipschitz continuity for minimizers of the obstacle problem, were forced to deal with the relation between minima and extremals, in the sense of solutions to a corresponding variational inequality. In that specific situation, this problem has been solved thanks to a suitable higher differentiability result and imposing a smallness condition on the gap between the coercivity and the growth exponent of the lagrangian. Indeed, a direct derivation of such a relation can be obtained in a trivial way only if the gap between the growth and the ellipticity exponent satisfies a suitable smallness condition.

On the other hand, relying on techniques of convex analysis, Scheven and Schmidt $[21,22]$ investigated the Dirichlet minimization problem for the total variation and the area functional with one-sided obstacle. The main point is that they were able to identify certain dual maximization problems for bounded divergence-measure fields and to establish duality formulas and pointwise relations between (generalized) $B V$ minimizers and dual maximizers. Their results are very general and apply to very general obstacle, such as $B V$ obstacles and thin obstacles; the proof of their results crucially depend on a new version of Anzellotti type pairing which involves general divergence measure fields and specific representative of $B V$ functions, by employing several fine results on capacities and one-sided approximation.

This framework seems to be the right one in order to extend the results in [2] to very general obstacle problems, as long as, by means of the Anzellotti pairings we are able to express the natural counterpart of the variational inequality in this very general setting, which will reduce to the usual one once we have the right summability for the functions involved.

We also investigated the same problem for obstacle in the Sobolev class $W_{\text {loc }}^{2, q}(\Omega)$ and imposing a smallness condition on the gap $\frac{q}{p}$. In this case, we show that the solutions to the obstacle problem locally belong to $W^{1, q}(\Omega)$ and therefore they satisfy the corresponding variational inequality.

Our paper is organized as follows: in Section 2 we state the main results of the paper while Section 3 contains some notations and preliminary results. The proof of the main results are contained in Section 4 (proof of Theorem 2.1) and Section 5 (proof of Theorem 2.2). 


\section{Statement of the main results}

Let us consider a class of variational obstacle problems of the form

$$
\min \left\{\int_{\Omega} F(D z): z \in \mathbb{K}_{\psi}^{F}(\Omega)\right\}
$$

where $\Omega$ is a bounded open set of $\mathbb{R}^{n}, n \geq 2$. The integrand $F: \mathbb{R}^{n} \rightarrow \mathbb{R}$ is $\mathcal{C}^{1}$ and satisfies the following growth and strict convexity assumptions:

$$
\begin{aligned}
\ell|\xi|^{p} & \leq F(\xi) \leq L\left(1+|\xi|^{q}\right), \\
\nu\left|V_{p}(\xi)-V_{p}(\eta)\right|^{2} & \leq F(\xi)-F(\eta)-\left\langle F^{\prime}(\eta), \xi-\eta\right\rangle
\end{aligned}
$$

for all $\xi, \eta \in \mathbb{R}^{n}$, for $0<\ell<L, \nu>0$ and $1<p \leq q<\infty$ and where we used the customary notation

$$
V_{p}(\xi)=\left(1+|\xi|^{2}\right)^{\frac{p-2}{4}} \xi
$$

The function $\psi: \Omega \rightarrow[-\infty,+\infty)$ is called obstacle and will satisfy the following two assumptions

$$
\psi \text { is } \operatorname{Cap}_{q} \text {-quasi-continuous }
$$

where $q>1$ is the exponent appearing in assumption (H1). Moreover, the following compatibility condition with the boundary values is in force: there exists a function $g \in u_{0}+W_{0}^{1, q}(\Omega)$ with

$$
\psi \leq g^{*} \operatorname{Cap}_{q} \text {-a.e. on } \Omega .
$$

The class $\mathbb{K}_{\psi}^{F}(\Omega)$ is defined as

$$
\mathbb{K}_{\psi}^{F}(\Omega):=\left\{z \in u_{0}+W_{0}^{1, p}(\Omega): z^{*} \geq \psi \operatorname{Cap}_{q} \text {-a.e. in } \Omega, F(D z) \in L^{1}(\Omega)\right\},
$$

where $u_{0} \in W^{1, q}(\Omega)$ is a fixed boundary value.

The symbol $u^{*}$ denotes as usual the precise representative of the function $u$ in the spirit of Definition 3.3 (for more details we refer to Subsection 3.1). Assumption (2.4) means that we are assuming the existence of a function $g \in u_{0}+W_{0}^{1, q}(\Omega) \cap \mathbb{K}_{\psi}^{F}(\Omega)$, and therefore $\mathbb{K}_{\psi}^{F}(\Omega)$ is not empty.

To simplify the statement of our main result, we shall assume that the integrand $F$ satisfies a sort of $\Delta_{2}$ condition, i.e.

$$
F(\lambda \xi) \leq C(\lambda) F(\xi)
$$

for every real positive $\lambda>1$ and every $\xi \in \mathbb{R}^{n}$. Actually, without (H3), our result holds true supposing that $F\left(c D u_{0}\right) \in L^{1}(\Omega)$, for some constant $c>1$.

It is worth mentioning that if $G$ is a $\mathcal{C}^{1}$ function satisfying (H1) and (H2) with $p=q$, i.e. $G$ satisfies standard $p$-growth conditions, the minimization problem reduces to

$$
\min \left\{\int_{\Omega} G(D z): z \in \mathcal{K}_{\psi}(\Omega)\right\}
$$

where

$$
\mathcal{K}_{\psi}(\Omega):=\left\{z \in u_{0}+W_{0}^{1, p}(\Omega): z^{*} \geq \psi \text { Cap }_{p} \text {-a.e. in } \Omega\right\} .
$$

In this case, because of the standard growth conditions, it is well known that, if $u \in u_{0}+W_{0}^{1, p}(\Omega)$ is a solution to (2.6), then $u$ is also a solution to the corresponding 
variational inequality

$$
\int_{\Omega}\left\langle G^{\prime}(D u), D z-D u\right\rangle d x \geq 0
$$

for every $z \in \mathcal{K}_{\psi}(\Omega)$. This can be proved by observing that, since $\mathcal{K}_{\psi}(\Omega)$ is a convex set, the function $u+\varepsilon(z-u)=(1-\varepsilon) u+\varepsilon z \in \mathcal{K}_{\psi}(\Omega)$ is an admissible variation for each $0 \leq \varepsilon<1$. On the other hand, if $u \in \mathcal{K}_{\psi}(\Omega)$ and $\varphi \geq 0$, with $\varphi \in \mathcal{C}_{0}^{\infty}(\Omega)$, then $u+\varphi \in \mathcal{K}_{\psi}(\Omega)$ and thus, if $u$ is a solution to (2.6), then also the following inequality holds

$$
\int_{\Omega}\left\langle G^{\prime}(D u), D \varphi\right\rangle d x \geq 0
$$

for all $\varphi \in \mathcal{C}_{0}^{\infty}(\Omega), \varphi \geq 0$ (see [14]).

Our goal is to show that, if we assume (2.3) and (2.4), the solutions to obstacle problems with non standard growth conditions solve a natural counterpart of the usual variational inequality, expressed in terms of duality formulation of the obstacle problem, involving the minimizers and the corresponding dual maximizers, namely (2.12) below. This formula is based on a new version of the Anzellotti type pairing [1] and relies on fine properties of capacities and one-sided approximation. This allows us to take into account the very general nature of the obstacle considered in our paper. More precisely the main result of the manuscript can be stated as follows.

Theorem 2.1. Let $F: \mathbb{R}^{n} \rightarrow \mathbb{R}$ be a $\mathcal{C}^{1}$ function satisfying (H1), (H2) and (H3). Assume moreover that (2.3) and (2.4) hold true. If $u \in \mathbb{K}_{\psi}^{F}(\Omega)$ is the solution to the obstacle problem (2.1), then

$$
F^{*}\left(F^{\prime}(D u)\right) \in L^{1}(\Omega), \quad\left\langle F^{\prime}(D u), D u\right\rangle \in L^{1}(\Omega)
$$

and

$$
\operatorname{div} F^{\prime}(D u) \leq 0
$$

in the distributional sense. Moreover, it holds the following

$$
\int_{\Omega} F(D u) d x=\llbracket F^{\prime}(D u), \psi \rrbracket_{u_{0}}(\bar{\Omega})-\int_{\Omega} F^{*}\left(F^{\prime}(D u)\right) d x
$$

Here $F^{*}$ denotes the polar, or Fenchel conjugate, of the convex continuous function $F$, introduced in (3.1) of Subsection 2.4, while the symbol $\llbracket F^{\prime}(D u), \psi \rrbracket_{u_{0}}(\bar{\Omega})$ inspired by the Anzellotti pairing is defined in Subsection 3.5. Hence, in view of Theorem 2.1, $u$ in particular solves the corresponding variational inequality and $F^{\prime}(D u) \in L^{q^{\prime}}\left(\Omega ; \mathbb{R}^{n}\right)$ with $q^{\prime}=\frac{q}{q-1}$.

It is worth noting that, under our assumptions, $F(\xi)$ needs not to depend on the length of $\xi$ nor to be the sum of its components $\xi_{i}$. Indeed, as an example of $F(\xi)$ satisfying our assumptions we have the following

$$
F(\xi)=\left|\xi_{1}-\xi_{2}\right|^{q}+\left|\xi_{1}+\xi_{2}\right|^{p} \log ^{\alpha}\left(1+\left|\xi_{1}\right|\right), \quad \xi \in \mathbb{R}^{2}, \alpha \in \mathbb{R},
$$

with $2 \leq p \leq q$ (constructed in [7]) .

In case the gap $\frac{q}{p}$ satisfies a suitable smallness assumption and if the obstacle $\psi \in W_{\text {loc }}^{2, q}(\Omega)$, we are able to prove that the solution to problem

$$
\min \left\{\int_{\Omega} F(D z): z \in \mathcal{K}_{\psi}(\Omega)\right\},
$$


with $\mathcal{K}_{\psi}(\Omega)$ as in $(2.7)$, locally belongs to $W_{\text {loc }}^{1, q}(\Omega)$ and therefore it satisfies the corresponding variational inequality, without any regularity on the boundary datum $u_{0}$. More precisely, we have the following

Theorem 2.2. Let $F: \mathbb{R}^{n} \rightarrow \mathbb{R}$ be a $\mathcal{C}^{1}$ function satisfying (H1), (H2) and (H3). Assume that

$$
D \psi \in W_{\text {loc }}^{1, q}(\Omega)
$$

and let $u \in \mathcal{K}_{\psi}(\Omega)$ be the solution to the obstacle problem (2.13). If

$$
1<p \leq q<\frac{n p}{n-1}
$$

then

and

$$
F^{*}\left(F^{\prime}(D u)\right) \in L_{\mathrm{loc}}^{1}(\Omega), \quad\left\langle F^{\prime}(D u), D u\right\rangle \in L_{\mathrm{loc}}^{1}(\Omega)
$$

$$
\operatorname{div} F^{\prime}(D u) \leq 0
$$

locally, in the distributional sense and moreover $u \in W_{\text {loc }}^{1, q}(\Omega)$.

Remark 2.3. Note that, arguing as in [2], in case

$$
\frac{n p}{n-1} \leq q<p^{*}
$$

and $D \psi \in W_{\text {loc }}^{1, q}(\Omega)$, then the solution $u$ to the obstacle problem (2.6) belongs to $W_{\text {loc }}^{1, r}(\Omega)$ for all $r<\bar{p}$ being

$$
\bar{p}:=\frac{n p}{n-\frac{p}{p-1}\left(1-n\left(\frac{1}{p}-\frac{1}{q}\right)\right)} .
$$

This result prevents the Lavrentiev phenomenon that may occur in the case of anisotropic growth conditions.

Let us mention a few words about the techniques employed. Our Lagrangian $F$ has been suitably approximated by strictly convex and uniformly elliptic integrands $F_{k}$, in order to facilitate a systematic use of the dual problems, in the sense of Convex Analysis. The minimizers of $F_{k}$, say $u_{k}$, strongly converge in $W^{1, p}$ to the minimizer $u$ of (2.1) and to every such minimizer $u_{k}$ we can associate the solutions of certain dual maximization problems for divergence-measure fields.

Next, we establish duality formulas and pointwise relations between minimizers and dual maximizers that are preserved in passing to the limit. Such estimates will provide conditions in order for the variational inequality, in the form of (2.12), to hold for a constrained minimizer. The statement and the proofs of our results, that are the counterpart of those in [2] concerning the unconstrained setting, rely on a suitable version of Anzellotti type pairing which involve general divergence-measure fields and specific representation of Sobolev functions, and which reduces to integration by part formula once the correct summability is required on the fields involved.

\section{Notations and preliminary results}

In this paper we shall denote by $C$ or $c$ a general positive constant that may vary on different occasions, even within the same line of estimates. Relevant dependencies will be suitably emphasized using parentheses or subscripts. In what follows, $B(x, r)=B_{r}(x)=\left\{y \in \mathbb{R}^{n}:|y-x|<r\right\}$ will denote the ball centered at $x$ of radius $r$. We shall omit the dependence on the center and on the radius when no confusion arises. 
For the auxiliary function $V_{p}$, introduced in (2.2), we recall the following estimate (see the proof of [12, Lemma 8.3]):

Lemma 3.1. Let $1<p<\infty$. There exists a constant $c=c(n, p)>0$ such that

$$
c^{-1}\left(1+|\xi|^{2}+|\eta|^{2}\right)^{\frac{p-2}{2}} \leq \frac{\left|V_{p}(\xi)-V_{p}(\eta)\right|^{2}}{|\xi-\eta|^{2}} \leq c\left(1+|\xi|^{2}+|\eta|^{2}\right)^{\frac{p-2}{2}}
$$

for any $\xi, \eta \in \mathbb{R}^{n}, \xi \neq \eta$.

3.1. Capacities and quasi (semi)continuity. For $p \in[1, \infty)$ the $p$-capacity of a set $E \subset \Omega$ can be defined by

$$
\begin{aligned}
\operatorname{Cap}_{p}(E):=\inf \{ & \int_{\Omega}\left(|u|^{p}+|D u|^{p}\right) d x: u \in W^{1, p}(\Omega), u \geq 1 \text { a.e. on an open } \\
& \text { neighbourhood of } E\} .
\end{aligned}
$$

With this definition, we say that a property holds $\operatorname{Cap}_{p}$-a.e. on a set $U$ in $\Omega$ if it holds on $U \backslash E$ with some subset $E$ of $U$ such that $\operatorname{Cap}_{p}(E)=0$. It is clear from the definition that a set with zero $p$-capacity is negligible for the Lebesgue measure, therefore a $\operatorname{Cap}_{p}$-a.e. requirement is stronger than the corresponding $\mathcal{L}^{n}$ a.e. requirement, where $\mathcal{L}^{n}$ is the $n$-dimensional Lebesgue measure. For more details on this topic we refer to the monographs $[11,14]$.

With the notion of capacity, we recall the concept of (semi)continuity up to small sets.

Definition 3.2. For any $p \in[1, \infty)$, we say that a $\operatorname{Cap}_{p}$-a.e. defined, real-valued function $\Psi$ on an open subset $\Omega$ of $\mathbb{R}^{n}$ is Cap $_{p}$-quasi upper semicontinuous on $\Omega$ if for every $\varepsilon>0$ there exists an open subset $E$ of $\Omega$ with $\operatorname{Cap}_{p}(E)<\varepsilon$ such that the restriction of $\Psi$ to $\Omega \backslash E$ is everywhere defined and upper semicontinuous. In a similar way we can define the corresponding concept of $\mathrm{Cap}_{p}$-quasi lower semiconti-

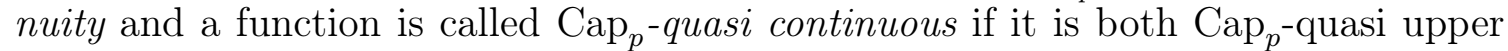
semicontinuous and $\mathrm{Cap}_{p}$-quasi lower semicontinuous.

Definition 3.3. Assume that $f \in L^{1}(\Omega)$. Then

$$
f^{*}(x)= \begin{cases}\lim _{r \rightarrow 0} f_{B(x, r)} f(y) d y & \text { if this limit exists, } \\ 0 & \text { otherwise }\end{cases}
$$

is the precise representative of $f$.

We remark that if $f, g \in L_{\text {loc }}^{1}(\Omega)$ with $f=g \mathcal{L}^{n}$-a.e., then $f^{*}=g^{*}$ for all points $x \in \Omega$. In view of the Lebesgue-Besicovith Differentiation Theorem with $\mu=\mathcal{L}^{n}$, $\lim _{r \rightarrow 0} f_{B(x, r)} f(y) d y$ exists $\mathcal{L}^{n}$-a.e.. Moreover the following result holds.

Theorem 3.4. Suppose that $f \in W^{1, p}(\Omega), 1 \leq p<n$.

(i) There exists a Borel set $E \subset \mathbb{R}^{n}$ such that

$$
\operatorname{Cap}_{p}(E)=0
$$

and

$$
\lim _{r \rightarrow 0} f_{B(x, r)} f(y) d y=f^{*}(x)
$$

exists for each $x \in \Omega \backslash E$. 
(ii) Moreover,

$$
\lim _{r \rightarrow 0} f_{B(x, r)}\left|f(y)-f^{*}(x)\right|^{p^{*}} d y=0
$$

for each $x \in \Omega \backslash E$.

(iii) The precise representative $f^{*}$ is $\mathrm{Cap}_{p}$-quasicontinuous.

We conclude this subsection recalling the following lemma.

Lemma 3.5. (Strong convergence in $W^{1,1}$ implies convergence $\mathcal{H}^{n-1}$-a.e.) Suppose that $u_{k}$ converges to $u$ strongly in $W^{1,1}(\Omega)$. Then there exists a subsequence $u_{k_{l}}^{*}$ that converges $\mathcal{H}^{n-1}$-a.e. on $\Omega$ to $u^{*}$.

3.2. Besov spaces. Let us recall that, for every function $f: \mathbb{R}^{n} \rightarrow \mathbb{R}$ the finite difference operator is defined by

$$
\tau_{s, h} f(x)=f\left(x+h e_{s}\right)-f(x)
$$

where $h \in \mathbb{R}, e_{s}$ is the unit vector in the $x_{s}$ direction and $s \in\{1, \ldots, n\}$. Let $1 \leq p<\infty$ and $0<\alpha<1$. The Besov space $B_{p, \infty}^{\alpha}\left(\mathbb{R}^{n}\right)$ consists of the function $v \in L^{p}\left(\mathbb{R}^{n}\right)$ such that

$$
[v]_{\dot{B}_{p, \infty}^{\alpha}\left(\mathbb{R}^{n}\right)}=\sup _{h \in \mathbb{R}^{n}}\left(\int_{\mathbb{R}^{n}} \frac{|v(x+h)-v(x)|^{p}}{|h|^{\alpha p}} d x\right)^{\frac{1}{p}}<\infty .
$$

One can simply take supremum over $|h| \leq \delta$ and obtain an equivalent norm. By construction, $B_{p, \infty}^{\alpha}\left(\mathbb{R}^{n}\right) \subset L^{p}\left(\mathbb{R}^{n}\right)$.

Given a domain $\Omega \subset \mathbb{R}^{n}$, we say that $v$ belongs to the local Besov space $B_{p, \infty, \text { loc }}^{\alpha}$ if $\varphi v$ belongs to the global Besov space $B_{p, q}^{\alpha}\left(\mathbb{R}^{n}\right)$ whenever $\varphi$ belongs to the class $\mathcal{C}_{0}^{\infty}(\Omega)$ of smooth functions with compact support contained in $\Omega$.

We also have the following embedding theorem that relates Sobolev or Lebesgue and Besov spaces, that can be deduced with the arguments of [23, Section 30-32].

Theorem 3.6. Let $\Omega \subset \mathbb{R}^{n}$. The continuous embedding

$$
B_{q, \infty, \mathrm{loc}}^{\alpha}(\Omega) \hookrightarrow L_{\mathrm{loc}}^{t}(\Omega)
$$

holds for all $t<\frac{n q}{n-\alpha q}$ provided $\alpha \in(0,1), q>1, \alpha q<n$ and the embedding

$$
W_{\mathrm{loc}}^{1, p}(\Omega) \hookrightarrow B_{q, \infty, \mathrm{loc}}^{\alpha}(\Omega)
$$

holds, provided $1<p<q<+\infty$ and $\alpha=1-n\left(\frac{1}{p}-\frac{1}{q}\right)>0$.

3.3. Some approximation results. Now, we state a useful approximation lemma whose proof can be found in [2, Proposition 3.1] and that will be needed in the sequel.

Lemma 3.7. Let $F: \mathbb{R}^{n} \rightarrow \mathbb{R}$ be a $\mathcal{C}^{1}$ function satisfying assumptions (H1)$(\mathrm{H} 2)$. Then there exists a sequence $\left(F_{k}\right)$ of $\mathcal{C}^{1}$ functions $F_{k}: \mathbb{R}^{n} \rightarrow \mathbb{R}$, monotonically convergent to $F$, such that

(I) for every $\xi \in \mathbb{R}^{n}$, and for every $k_{1}<k_{2}$, it holds

$$
F_{k_{1}}(\xi) \leq F_{k_{2}}(\xi) \leq F(\xi)
$$

(II) for every $\xi \in \mathbb{R}^{n}$, we have

$$
c(p, \nu)\left|V_{p}(\xi)-V_{p}(\eta)\right|^{2} \leq F_{k}(\xi)-F_{k}(\eta)-\left\langle F_{k}^{\prime}(\eta), \xi-\eta\right\rangle
$$


(III) for every $\xi \in \mathbb{R}^{n}$, there exist constants $L_{0}, L_{1}$, independent of $k$, and $\bar{L}_{1}$, depending on $k$, such that

$$
\begin{aligned}
L_{0}\left(|\xi|^{p}-1\right) & \leq F_{k}(\xi) \leq L_{1}(1+|\xi|)^{q}, \\
F_{k}(\xi) & \leq \bar{L}_{1}(k)(1+|\xi|)^{p},
\end{aligned}
$$

(IV) if $\xi_{k} \rightarrow \xi$, then $F_{k}{ }^{\prime}\left(\xi_{k}\right) \rightarrow F^{\prime}(\xi)$ locally uniformly.

Actually, a careful inspection of the proof of [2, Proposition 3.1] reveals that there exists a sequence $\mu_{k} \in \mathbb{R}$ such that

$$
\lim _{k \rightarrow \infty} \mu_{k}=0
$$

and

$$
F_{k}(\xi) \geq L_{0}|\xi|^{p}-\mu_{k} \quad \text { for every } k \in \mathbb{N} .
$$

In the sequel, we shall also use the following

Lemma 3.8. Let $\psi$ be $\operatorname{Cap}_{p}$-quasicontinuous and let $u_{0} \in W^{1, p}(\Omega)$, with $p \geq 1$. Suppose that there exists $g \in u_{0}+W_{0}^{1, p}(\Omega)$ such that

$$
g^{*}(x) \geq \psi(x) \quad \operatorname{Cap}_{p} \text {-a.e. in } \Omega .
$$

Then there exists a non increasing sequence of functions $\psi_{k} \in u_{0}+W_{0}^{1, p}(\Omega)$ such that

$$
\psi_{k}^{*} \rightarrow \psi \quad \mathrm{Cap}_{p} \text {-a.e. in } \Omega .
$$

For the proof, we refer to [22, Lemma 2.19] which is a suitable version for our purposes of [9, Lemma 1.5].

3.4. Harmonic extension of Sobolev functions. Let us fix a ball $B_{R} \subset \mathbb{R}^{n}$ and consider the following Dirichlet problem

$$
\begin{cases}\Delta h=0 & \text { in } B_{R}, \\ h=f & \text { on } \partial B_{R},\end{cases}
$$

where $f \in W^{1, p}\left(\partial B_{R}\right)$. Browder-Minty Theorem implies that problem (D) admits a unique solution $u \in W^{1, p}\left(B_{R}\right)$ and we can define the solution operator

$$
\mathcal{S}_{\Delta}: f \in W^{1, p}\left(\partial B_{R}\right) \mapsto u \in W^{1, p}\left(B_{R}\right) .
$$

We shall use the following particular case of [4, Theorem 4.1]:

Theorem 3.9. For $1<p \leq q \leq \frac{p n}{n-1}$, it holds that

$$
\left\|D \mathcal{S}_{\Delta}(f)\right\|_{L^{q}\left(B_{R} ; \mathbb{R}^{n}\right)} \leq c(n, p, q)\|D f\|_{L^{p}\left(\partial B_{R} ; \mathbb{R}^{n-1}\right)}\|f\|_{L^{p}\left(\partial B_{R}\right)} .
$$

It is worth mentioning that previous result is well known, but it is difficult to find an explicit proof and this is the reason why we refer to [4].

3.5. Dual formulation of the obstacle problem. This section is devoted to establish the dual formulation of obstacle problems with standard growth conditions, extending classical ideas of Kohn and Temam [15] and Anzellotti [1] and following [22]. For the readers' convenience we recall a few key results about convex duality here and we refer to [10, Chapter 1] for details.

Given a convex continuous function $F: \mathbb{R}^{n} \rightarrow \mathbb{R}$, its polar (or Fenchel conjugate) is defined by

$$
F^{*}(\zeta):=\sup _{\xi \in \mathbb{R}^{n}}(\langle\zeta, \xi\rangle-F(\xi)) \quad \forall \zeta \in \mathbb{R}^{n}
$$


The function $F^{*}: \mathbb{R}^{n} \rightarrow \mathbb{R}$ is convex and, if $F$ satisfies assumption $(\mathrm{H} 1), F^{*}$ has $\left(q^{\prime}, p^{\prime}\right)$ growth, where $p^{\prime}$ and $q^{\prime}$ are the Hölder conjugate exponents of $p, q$ respectively, i.e. there exist constants $c(L), c(\ell)$ such that

$$
c(L)|\zeta|^{q^{\prime}}-L \leq F^{*}(\zeta) \leq c(\ell)|\zeta|^{p^{\prime}} \quad \forall \zeta \in \mathbb{R}^{n} .
$$

One can check that the bipolar integrand $F^{* *}:=\left(F^{*}\right)^{*}$ equals $F$ at $\xi$ if and only if $F$ is lower semicontinuous and convex at $\xi$, as it is the case here.

From the definition of polar function directly follows the Young-type (or Fenchel) inequality

$$
\langle\zeta, \xi\rangle \leq F^{*}(\zeta)+F(\xi)
$$

for all $\zeta, \xi \in \mathbb{R}^{n}$. Notice that, for a given $\xi$, we have equality in (3.2) precisely for $\zeta \in \partial F^{* *}(\xi)$, the subgradient of $F^{* *}$ at $\xi$. In particular, when $F$ is $\mathcal{C}^{1}$, for every $\xi \in \mathbb{R}^{n}$, we have equality in (3.2) precisely for $\zeta=F^{\prime}(\xi)$. Actually, it holds the following

$$
F(\xi)+F^{*}\left(F^{\prime}(\xi)\right)=\left\langle F^{\prime}(\xi), \xi\right\rangle,
$$

for every $\xi \in \mathbb{R}^{n}$. Indeed, the convexity of $F$, since $F \in \mathcal{C}^{1}$, gives

$$
F(\xi) \geq F(\eta)+\left\langle F^{\prime}(\eta), \xi-\eta\right\rangle \quad \forall \xi, \eta \in \mathbb{R}^{n},
$$

which is equivalent to

$$
\left\langle F^{\prime}(\eta), \eta\right\rangle-F(\eta) \geq\left\langle F^{\prime}(\eta), \xi\right\rangle-F(\xi) \quad \forall \xi, \eta \in \mathbb{R}^{n} .
$$

From this we deduce that

$$
\left\langle F^{\prime}(\eta), \eta\right\rangle-F(\eta) \geq \sup _{\xi \in \mathbb{R}^{n}}\left[\left\langle F^{\prime}(\eta), \xi\right\rangle-F(\xi)\right]=F^{*}\left(F^{\prime}(\eta)\right),
$$

by the definition of $F^{*}(\zeta)$ at (3.1). Thus

$$
\left\langle F^{\prime}(\eta), \eta\right\rangle \geq F(\eta)+F^{*}\left(F^{\prime}(\eta)\right)
$$

which obviously gives equality in (3.2), i.e. (3.3).

Now, we consider for any $p>1$

$$
S_{-}^{p^{\prime}}(\Omega)=\left\{\sigma \in L^{p^{\prime}}(\Omega): \operatorname{div} \sigma \leq 0 \text { in } \mathcal{D}^{\prime}(\Omega)\right\},
$$

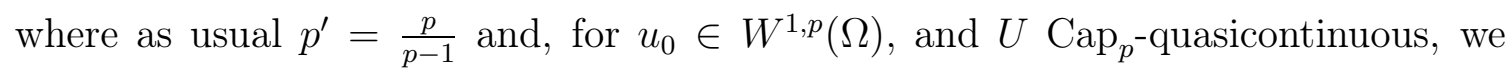
introduce a measure $\llbracket \sigma, U \rrbracket_{u_{0}}$ on $\bar{\Omega}$ by setting

$$
\llbracket \sigma, U \rrbracket_{u_{0}}(\bar{\Omega})=\int_{\Omega}\left(U-u_{0}^{*}\right) d(-\operatorname{div} \sigma)+\int_{\Omega}\left\langle\sigma, D u_{0}\right\rangle d x
$$

where we recall that the symbol $u^{*}$ denotes the precise representative of $u$. It is known that for $\sigma \in S_{-}^{p^{\prime}}(\Omega)$, the distribution $\operatorname{div} \sigma$ is a non positive Radon measure that vanishes on set with $p$-capacity zero (see [22, Section 4]). Since the precise representative is a function $\mathrm{Cap}_{p}$-a.e. defined, the duality pairing defined above is meaningful in this setting.

For $\tilde{u} \in u_{0}+W_{0}^{1, p}(\Omega)$, the quantity $\llbracket \sigma, \tilde{u} \rrbracket_{u_{0}}(\bar{\Omega})$ corresponds to the integral of the function $\langle\sigma, D \tilde{u}\rangle \in L^{1}(\Omega)$ as it follows from the well known integration by parts formula

$$
\int_{\Omega} \varphi d(-\operatorname{div} \sigma)=\int_{\Omega}\langle\sigma, D \varphi\rangle d x
$$

that holds true for every $\varphi \in W_{0}^{1, p}(\Omega)$. 
The dual formulation of obstacle problems under standard growth conditions is contained in the following:

Theorem 3.10. Let $G: \mathbb{R}^{n} \rightarrow \mathbb{R}$ be a $\mathcal{C}^{1}$, strictly convex function satisfying

$$
\ell_{p}\left(|\xi|^{p}-1\right) \leq G(\xi) \leq L_{p}\left(1+|\xi|^{p}\right)
$$

for all $\xi \in \mathbb{R}^{n}$, for constants $0<\ell_{p} \leq L_{p}$ and an exponent $p>1$. If $u_{0} \in W^{1, p}(\Omega)$, $\psi$ satisfies (2.3) and (2.4) with $p=q$, then

$$
\min _{v \in \mathcal{K}_{\psi}(\Omega)} \int_{\Omega} G(D v) d x=\max _{\sigma \in S_{-}^{p^{\prime}}(\Omega)}\left(\llbracket \sigma, \psi \rrbracket_{u_{0}}(\bar{\Omega})-\int_{\Omega} G^{*}(\sigma) d x\right)
$$

where $S_{-}^{p^{\prime}}(\Omega), \llbracket \sigma, \psi \rrbracket_{u_{0}}$ and $\mathcal{K}_{\psi}(\Omega)$ are defined in (3.4), (3.5) and (2.7) respectively. If, moreover, $u \in \mathcal{K}_{\psi}(\Omega)$ is the solution to (2.6), then

$$
\int_{\Omega} G(D u) d x=\llbracket G^{\prime}(D u), \psi \rrbracket_{u_{0}}(\bar{\Omega})-\int_{\Omega} G^{*}\left(G^{\prime}(D u)\right) d x .
$$

Proof. Let us consider an arbitrary vector field $\sigma \in S_{-}^{p^{\prime}}(\Omega)$ and a function $v \in \mathcal{K}_{\psi}(\Omega)$. Since $-\operatorname{div} \sigma$ is a non-negative Radon measure and $v^{*} \geq \psi$ Cap $_{p}$-a.e. in $\Omega$, we have

$$
\int_{\Omega}\left(v^{*}-\psi\right) d(-\operatorname{div} \sigma) \geq 0
$$

By the definition at (3.5) and by using (3.9), we infer that

$$
\begin{aligned}
\llbracket \sigma, \psi \rrbracket_{u_{0}}(\bar{\Omega}) & =\int_{\Omega}\left(\psi-u_{0}^{*}\right) d(-\operatorname{div} \sigma)+\int_{\Omega}\left\langle\sigma, D u_{0}\right\rangle d x \\
& =\int_{\Omega}\left(\psi-v^{*}+v^{*}-u_{0}^{*}\right) d(-\operatorname{div} \sigma)+\int_{\Omega}\left\langle\sigma, D u_{0}\right\rangle d x \\
& \leq \int_{\Omega}\left(v^{*}-u_{0}^{*}\right) d(-\operatorname{div} \sigma)+\int_{\Omega}\left\langle\sigma, D u_{0}\right\rangle d x .
\end{aligned}
$$

Since $v, u_{0} \in W^{1, p}(\Omega)$ and $v=u_{0}$ on $\partial \Omega$, we can use (3.6) in the first integral of the last line of previous formula, thus getting

$$
\begin{aligned}
\llbracket \sigma, \psi \rrbracket_{u_{0}}(\bar{\Omega}) & =\int_{\Omega}\left\langle\sigma, D v-D u_{0}\right\rangle d x+\int_{\Omega}\left\langle\sigma, D u_{0}\right\rangle d x \\
& =\int_{\Omega}\langle\sigma, D v\rangle d x \leq \int_{\Omega} G(D v) d x+\int_{\Omega} G^{*}(\sigma) d x
\end{aligned}
$$

by the Young's type inequality at (3.2). This entails that

$$
\int_{\Omega} G(D v) d x \geq \llbracket \sigma, \psi \rrbracket_{u_{0}}(\bar{\Omega})-\int_{\Omega} G^{*}(\sigma) d x
$$

and thus, passing to the minimum in $v$ in the left hand side and to the maximum in $\sigma$ in the right hand side of previous inequality, we get

$$
\min _{v \in \mathcal{K}_{\psi}(\Omega)} \int_{\Omega} G(D v) d x \geq \max _{\sigma \in S_{-}^{p^{\prime}}(\Omega)}\left(\llbracket \sigma, \psi \rrbracket_{u_{0}}(\bar{\Omega})-\int_{\Omega} G^{*}(\sigma) d x\right) .
$$


In order to prove the reverse inequality, we start from (3.3) with the choice $F=G$ and $\xi=D u$, being $u \in \mathcal{K}_{\psi}$ a solution to (2.6). Integrating over $\Omega$ we have that

$$
\begin{aligned}
\int_{\Omega} G(D u) d x & =\int_{\Omega}\left\langle G^{\prime}(D u), D u\right\rangle d x-\int_{\Omega} G^{*}\left(G^{\prime}(D u)\right) d x \\
& =\int_{\Omega}\left\langle G^{\prime}(D u), D u-D u_{0}\right\rangle d x+\int_{\Omega}\left\langle G^{\prime}(D u), D u_{0}\right\rangle d x-\int_{\Omega} G^{*}\left(G^{\prime}(D u)\right) d x .
\end{aligned}
$$

Accordingly to the terminology used so far, we set $\sigma:=G^{\prime}(D u)$ and use again (3.6) to deduce that

$$
\begin{aligned}
\int_{\Omega} G(D u) d x= & \int_{\Omega}\left\langle\sigma, D u-D u_{0}\right\rangle d x+\int_{\Omega}\left\langle\sigma, D u_{0}\right\rangle d x-\int_{\Omega} G^{*}(\sigma) d x \\
= & \int_{\Omega}\left(u^{*}-u_{0}^{*}\right) d(-\operatorname{div} \sigma)+\int_{\Omega}\left\langle\sigma, D u_{0}\right\rangle d x-\int_{\Omega} G^{*}(\sigma) d x \\
= & \int_{\Omega}\left(\psi-\psi+u^{*}-u_{0}^{*}\right) d(-\operatorname{div} \sigma)+\int_{\Omega}\left\langle\sigma, D u_{0}\right\rangle d x-\int_{\Omega} G^{*}(\sigma) d x \\
= & \int_{\Omega}\left(\psi-u_{0}^{*}\right) d(-\operatorname{div} \sigma)-\int_{\Omega}\left(\psi-u^{*}\right) d(-\operatorname{div} \sigma) \\
& +\int_{\Omega}\left\langle\sigma, D u_{0}\right\rangle d x-\int_{\Omega} G^{*}(\sigma) d x .
\end{aligned}
$$

Now, since $u \in u_{0}+W^{1, p}(\Omega)$ and $u^{*} \geq \psi$ as long as $u$ is the solution to our obstacle problem, we can use Lemma 3.8 to construct a non increasing sequence $\psi_{k} \in u_{0}+W_{0}^{1, p}(\Omega)$ such that

$$
\psi_{k}^{*} \rightarrow \psi \quad \mathrm{Cap}_{p} \text {-a.e. in } \Omega .
$$

Therefore,

$$
\psi_{k} \in \mathcal{K}_{\psi}(\Omega)
$$

and, since $u$ is a solution to (2.8), we have

$$
\int_{\Omega}\left\langle\sigma, D \psi_{k}-D u\right\rangle d x \geq 0 \quad \text { for every } k \in \mathbb{N} .
$$

Via the Monotone Convergence Theorem, passing to the limit in the previous inequality, we deduce that

$$
\begin{aligned}
\int_{\Omega}\left(\psi-u^{*}\right) d(-\operatorname{div} \sigma) & =\lim _{k \rightarrow \infty} \int_{\Omega}\left(\psi_{k}^{*}-u^{*}\right) d(-\operatorname{div} \sigma) \\
& =\lim _{k \rightarrow \infty} \int_{\Omega}\left\langle\sigma, D \psi_{k}-D u\right) d x \geq 0,
\end{aligned}
$$

where we used again (3.6). Inserting (3.12) in (3.11) we obtain

$$
\begin{aligned}
\int_{\Omega} G(D u) d x & \leq \int_{\Omega}\left(\psi-u_{0}^{*}\right) d(-\operatorname{div} \sigma)+\int_{\Omega}\left\langle\sigma, D u_{0}\right\rangle d x-\int_{\Omega} G^{*}(\sigma) d x \\
& \leq \llbracket \sigma, \psi \rrbracket_{u_{0}}(\bar{\Omega})-\int_{\Omega} G^{*}(\sigma) d x
\end{aligned}
$$


Summing up we have

$$
\begin{aligned}
\min _{v \in \mathcal{K}_{\psi}(\Omega)} \int_{\Omega} G(D v) d x & =\int_{\Omega} G(D u) d x \leq \llbracket \sigma, \psi \rrbracket_{u_{0}}(\bar{\Omega})-\int_{\Omega} G^{*}(\sigma) d x \\
& \leq \max _{\sigma \in S_{-}^{p^{\prime}}(\Omega)}\left\{\llbracket \sigma, \psi \rrbracket_{u_{0}}(\bar{\Omega})-\int_{\Omega} G^{*}(\sigma) d x\right\} .
\end{aligned}
$$

Combining previous estimate with (3.10), we establish (3.7) and, recalling that $\sigma=$ $G^{\prime}(D u)$, the equality at (3.8).

\section{Proof of Theorem 2.1}

In this section we shall establish the validity of the variational inequality associated to our obstacle problem, by using the duality theory and the approximation Lemma of the previous section. More precisely we are ready to give the

Proof of Theorem 2.1. For the sake of clarity we shall divide the proof in steps. In the first one, we shall use the approximation Lemma to construct a sequence of obstacle problems with standard growth conditions for which the dual problem is given by Theorem 3.10. In the second step, we prove that the sequence of approximating minimizers converges to the solution of problem (2.1), as well as the sequence of dual maximizers converges to a field whose divergence is a non positive Radon measure. Finally in Step 3 and 4 we establish the validity of the variational inequality.

Step 1. The approximation. Let $F_{k}$ be the sequence of functionals obtained applying Lemma 3.7 to the integrand $F$. We recall that $F_{k} \nearrow F$ and that $F_{k}$ are of class $\mathcal{C}^{1}$ and strictly convex, with $p$-growth.

Let $u_{k} \in \mathcal{K}_{\psi}(\Omega)$ be the solution to the obstacle problem

$$
\min _{w \in \mathcal{K}_{\psi}(\Omega)} \int_{\Omega} F_{k}(D w) d x
$$

and let

$$
\sigma_{k}:=F_{k}^{\prime}\left(D u_{k}\right) \in \mathcal{S}_{-}^{p^{\prime}}(\Omega)
$$

be the solution to the dual problem given by (3.7), i.e. $\sigma_{k}$ is such that

$$
\max _{\sigma \in S_{-}^{p^{\prime}}(\Omega)}\left\{\llbracket \sigma, \psi \rrbracket_{u_{0}}(\bar{\Omega})-\int_{\Omega} F_{k}^{*}(\sigma) d x\right\}=\llbracket \sigma_{k}, \psi \rrbracket_{u_{0}}(\bar{\Omega})-\int_{\Omega} F_{k}^{*}\left(\sigma_{k}\right) d x,
$$

where $F_{k}^{*}$ denotes the polar function of $F_{k}$. By (II) and (III) of Lemma 3.7, we are legitimate to apply Theorem 3.10 to each $F_{k}$. Therefore, from (3.8) with $G=F_{k}$, $u=u_{k}$ and $\sigma=\sigma_{k}$, we have that the following equality

$$
\int_{\Omega} F_{k}\left(D u_{k}\right) d x=\llbracket \sigma_{k}, \psi \rrbracket_{u_{0}}(\bar{\Omega})-\int_{\Omega} F_{k}^{*}\left(\sigma_{k}\right) d x
$$

holds for all $k \in \mathbb{N}$.

As long as $F_{k}$ satisfy a uniform $(p, q)$-growth condition, then, as already remarked in Section $3, F_{k}^{*}$ satisfy a uniform $\left(q^{\prime}, p^{\prime}\right)$-growth condition, and, since $F_{k}(\xi) \nearrow F(\xi)$, it is not difficult to check that $F_{k}^{*}(\zeta) \searrow F^{*}(\zeta)$ as $k \rightarrow \infty$, pointwise in $\zeta$. Furthermore, since $F_{k}$ satisfy standard growth conditions, we also have that $u_{k}$ solve the following variational inequality

$$
\int_{\Omega}\left\langle\sigma_{k}, D \varphi-D u_{k}\right\rangle d x \geq 0 \quad \forall \varphi \in \mathcal{K}_{\psi}(\Omega) \text { and } \forall k \in \mathbb{N} .
$$


Step 2. Passage to the limit. Our next purpose is to prove that $u_{k} \rightarrow u$ strongly in $W^{1, p}(\Omega)$, where $u$ is the solution to the obstacle problem at $(2.1)$.

First of all, we observe that

$$
\begin{aligned}
L_{0} \int_{\Omega}\left|D u_{k}\right|^{p} d x & \leq \int_{\Omega}\left[F_{k}\left(D u_{k}\right)+L_{0}\right] d x \leq \int_{\Omega}\left[F_{k}(D g)+L_{0}\right] d x \\
& \leq \int_{\Omega}\left[F(D g)+L_{0}\right] d x<+\infty,
\end{aligned}
$$

where we used the growth condition on $F_{k}$ expressed at (III) of Lemma 3.7, the minimality of $u_{k}$, the fact that, by virtue of assumption (2.4), we can use $g$ as test function and also that $F_{k} \nearrow F$. This tells us that the sequence $\left\{u_{k}\right\}_{k}$ is bounded in $W^{1, p}(\Omega)$. Then, by the reflexivity of $W^{1, p}(\Omega)$, it admits a subsequence weakly converging to some $v \in W^{1, p}(\Omega)$. We have that $v \in \mathcal{K}_{\psi}(\Omega)$ because $u_{k} \in \mathcal{K}_{\psi}(\Omega)$ and $\mathcal{K}_{\psi}(\Omega)$ is a convex closed set, therefore weakly closed.

Fix $k_{0} \in \mathbb{N}$, by the lower semicontinuity of $F_{k_{0}}$, we have

$$
\liminf _{k \rightarrow+\infty} \int_{\Omega} F_{k_{0}}\left(D u_{k}\right) d x \geq \int_{\Omega} F_{k_{0}}(D v) d x
$$

and the monotonicity of the sequence $F_{k}$ yields

$$
\int_{\Omega} F_{k_{0}}\left(D u_{k}\right) d x \leq \int_{\Omega} F_{k}\left(D u_{k}\right) d x
$$

for every $k>k_{0}$. Therefore

$$
\int_{\Omega} F_{k_{0}}(D v) d x \leq \liminf _{k \rightarrow+\infty} \int_{\Omega} F_{k}\left(D u_{k}\right) d x
$$

and since $F_{k} \nearrow F$, taking the limit as $k_{0} \rightarrow \infty$, we deduce, by the Monotone Convergence Theorem, that

$$
\liminf _{k \rightarrow+\infty} \int_{\Omega} F_{k}\left(D u_{k}\right) d x \geq \int_{\Omega} F(D v) d x .
$$

Remark that previous inequality implies

$$
\int_{\Omega} F(D v) d x \leq \liminf _{k \rightarrow+\infty} \int_{\Omega} F_{k}\left(D u_{k}\right) d x \leq \liminf _{k \rightarrow+\infty} \int_{\Omega} F_{k}(D g) \leq \int_{\Omega} F(D g) d x .
$$

Thus in particular it turns out that $v \in \mathbb{K}_{\psi}^{F}(\Omega)$ and so we can exploit the minimality of $u$ in the class $\mathbb{K}_{\psi}^{F}(\Omega)$ to finally end up with

$$
\liminf _{k \rightarrow+\infty} \int_{\Omega} F_{k}\left(D u_{k}\right) d x \geq \int_{\Omega} F(D v) d x \geq \int_{\Omega} F(D u) d x .
$$

On the other hand, by the minimality of $u_{k}$ we have

$$
\int_{\Omega} F_{k}\left(D u_{k}\right) d x \leq \int_{\Omega} F_{k}(D u) d x
$$

since $u \in \mathbb{K}_{\psi}(\Omega) \subset \mathcal{K}_{\psi}(\Omega)$. Using once more the Monotone Convergence Theorem, we get

$$
\limsup _{k \rightarrow+\infty} \int_{\Omega} F_{k}\left(D u_{k}\right) d x \leq \limsup _{k \rightarrow+\infty} \int_{\Omega} F_{k}(D u) d x=\int_{\Omega} F(D u) d x .
$$

By a direct comparison of (4.5) and (4.7) we deduce that

$$
\int_{\Omega} F_{k}\left(D u_{k}\right) d x \rightarrow \int_{\Omega} F(D u) d x=\int_{\Omega} F(D v) d x
$$


but the strict convexity of $F$ implies the uniqueness of the solutions and therefore $u=v$.

To deduce the strong convergence of $u_{k}$ towards $u$, we exploit (II) of Lemma 3.7 and (4.3) with $u$ in place of $\varphi$, namely

$$
\begin{aligned}
& c(p, \nu) \int_{\Omega}\left|V_{p}(D u)-V_{p}\left(D u_{k}\right)\right|^{2} d x \\
& \leq \int_{\Omega}\left(F_{k}(D u)-F_{k}\left(D u_{k}\right)-\left\langle F_{k}^{\prime}\left(D u_{k}\right), D u-D u_{k}\right\rangle\right) d x \\
& \leq \int_{\Omega}\left(F_{k}(D u)-F_{k}\left(D u_{k}\right)\right) d x \rightarrow 0
\end{aligned}
$$

as $k \rightarrow+\infty$, where in the last line we used (4.8) and the equality in (4.7). Therefore, by Lemma 3.1, we get

$$
c \int_{\Omega}\left|D u-D u_{k}\right|^{2}\left(1+|D u|^{2}+\left|D u_{k}\right|^{2}\right)^{\frac{p-2}{2}} d x \leq \int_{\Omega}\left|V_{p}(D u)-V_{p}\left(D u_{k}\right)\right|^{2} d x \rightarrow 0
$$

which entails the desired strong convergence

$$
u_{k} \rightarrow u \text { strongly in } W^{1, p}(\Omega) .
$$

Indeed, if $p \geq 2$, this follows from the trivial inequality

$$
\left|D u-D u_{k}\right|^{p} \leq\left|D u-D u_{k}\right|^{2}\left(1+|D u|^{2}+\left|D u_{k}\right|^{2}\right)^{\frac{p-2}{2}}
$$

while, for $1<p<2$, we may use Hölder's inequality with exponents $\frac{2}{p}$ and $\frac{2}{2-p}$ as follows

$$
\begin{aligned}
& \int_{\Omega}\left|D u-D u_{k}\right|^{p} d x \\
& =\int_{\Omega}\left|D u-D u_{k}\right|^{p}\left(1+|D u|^{2}+\left|D u_{k}\right|^{2}\right)^{\frac{p(p-2)}{4}}\left(1+|D u|^{2}+\left|D u_{k}\right|^{2}\right)^{\frac{p(2-p)}{4}} d x \\
& \leq\left(\int_{\Omega}\left|D u-D u_{k}\right|^{2}\left(1+|D u|^{2}+\left|D u_{k}\right|^{2}\right)^{\frac{(p-2)}{2}} d x\right)^{\frac{p}{2}}\left(\int_{\Omega}\left(1+|D u|^{2}+\left|D u_{k}\right|^{2}\right)^{\frac{p}{2}} d x\right)^{\frac{2-p}{2}} \\
& \leq C\left(\int_{\Omega}\left|D u-D u_{k}\right|^{2}\left(1+|D u|^{2}+\left|D u_{k}\right|^{2}\right)^{\frac{(p-2)}{2}} d x\right)^{\frac{p}{2}}
\end{aligned}
$$

where, in the last line, we used (4.4).

The use of (IV) of Lemma 3.7 for $\xi_{k}=D u_{k}$ and $\xi=D u$, yields that

$$
\sigma_{k}=F_{k}^{\prime}\left(D u_{k}\right) \rightarrow F^{\prime}(D u) \text { locally uniformly as } k \rightarrow \infty \text {. }
$$

It follows in particular that $F_{k}^{\prime}\left(D u_{k}\right) \rightarrow F^{\prime}(D u)$ in measure on $\Omega$ and so passing to the limit in the equality

$$
\left\langle\sigma_{k}, D u_{k}\right\rangle=F_{k}^{*}\left(\sigma_{k}\right)+F_{k}\left(D u_{k}\right)
$$

which has been deduced by (3.3) with $\xi=D u_{k}$ and $G=F_{k}$, we recover, with $\sigma=F^{\prime}(D u)$, the pointwise extremality relation

$$
\left\langle F^{\prime}(D u), D u\right\rangle=F^{*}\left(F^{\prime}(D u)\right)+F(D u) .
$$

Step 3. The validity of (2.10). Since by assumption (2.4), $g \in \mathbb{K}_{\psi}^{F}(\Omega) \subset \mathcal{K}_{\psi}(\Omega)$ we can use (4.3) with $g$ in place of $\varphi$, thus getting

$$
\int_{\Omega}\left\langle\sigma_{k}, D g-D u_{k}\right\rangle d x \geq 0 \quad \forall k \in \mathbb{N} .
$$


Therefore, integrating (4.9) over $\Omega$ and using previous inequality, we obtain

$$
\begin{aligned}
\int_{\Omega} F_{k}^{*}\left(\sigma_{k}\right) d x & =\int_{\Omega}\left\langle\sigma_{k}, D u_{k}\right\rangle d x-\int_{\Omega} F_{k}\left(D u_{k}\right) d x \\
& \leq \int_{\Omega}\left\langle\sigma_{k}, D g\right\rangle d x-\int_{\Omega} F_{k}\left(D u_{k}\right) d x \\
& =\frac{1}{2} \int_{\Omega}\left\langle\sigma_{k}, 2 D g\right\rangle d x-\int_{\Omega} F_{k}\left(D u_{k}\right) d x \\
& \leq \frac{1}{2} \int_{\Omega} F_{k}^{*}\left(\sigma_{k}\right) d x+\frac{1}{2} \int_{\Omega} F_{k}(2 D g)-\int_{\Omega} F_{k}\left(D u_{k}\right) d x
\end{aligned}
$$

Reabsorbing the first term in the right hand side by the left hand side, we get

$$
\frac{1}{2} \int_{\Omega} F_{k}^{*}\left(\sigma_{k}\right) d x \leq \frac{1}{2} \int_{\Omega} F(2 D g) d x-\int_{\Omega} F_{k}\left(D u_{k}\right) d x \leq C \int_{\Omega} F(D g) d x
$$

by (H3) and (4.4).

Recalling that $F_{k}^{*} \searrow F^{*}$, from (4.11) we also have that

$$
\int_{\Omega} F^{*}\left(\sigma_{k}\right) d x \leq C \int_{\Omega} F(D g) d x
$$

Since we already observed that $\sigma_{k} \rightarrow F^{\prime}(D u)$ a.e., by Fatou's Lemma and by previous estimate

$$
\int_{\Omega} F^{*}\left(F^{\prime}(D u)\right) d x \leq \liminf _{k \rightarrow+\infty} \int_{\Omega} F^{*}\left(\sigma_{k}\right) d x \leq C \int_{\Omega} F(D g) d x
$$

Thus

$$
F^{*}\left(F^{\prime}(D u)\right) \in L^{1}(\Omega)
$$

Whence, by (4.10), we also have

$$
\left\langle F^{\prime}(D u), D u\right\rangle \in L^{1}(\Omega)
$$

since $F(D u) \in L^{1}(\Omega)$ by the definition of minimizer.

Step 4 . The validity of the variational inequality. For this purpose, we note that in view of the $\left(q^{\prime}, p^{\prime}\right)$-growth of $F^{*}(\sigma)$ and of $F_{k}^{*}\left(\sigma_{k}\right)$, previous inequality and (4.11),

$$
\int_{\Omega}\left|F^{\prime}(D u)\right|^{q^{\prime}} d x \leq \limsup _{k \rightarrow+\infty} \int_{\Omega}\left|\sigma_{k}\right|^{q^{\prime}} \leq C \int_{\Omega} F(D g) d x
$$

Therefore

$$
\sigma_{k} \rightarrow \sigma \quad \text { weakly in } L^{q^{\prime}}(\Omega)
$$

and by the convergence of $\sigma_{k}$ to $\sigma$ in measure, we also have

$$
\sigma_{k} \rightarrow \sigma \text { strongly in } L^{r}(\Omega) \text { for every } r<q^{\prime} \text {; }
$$

thus also $\sigma_{k} \rightarrow \sigma$ a.e. up to a subsequence. The minimality of $u_{k}$ yields the validity of the following variational inequality

$$
\int_{\Omega}\left\langle\sigma_{k}, D \eta\right\rangle d x \geq 0 \quad \text { for all } \eta \in C_{0}^{\infty}(\Omega), \eta \geq 0
$$

and so, by the weak convergence of $\sigma_{k}$ to $\sigma$ in $L^{q^{\prime}}(\Omega)$, passing to the limit as $k \rightarrow \infty$ in previous inequality, also

$$
\int_{\Omega}\langle\sigma, D \eta\rangle d x \geq 0 \quad \text { for all } \eta \in C_{0}^{\infty}(\Omega), \eta \geq 0
$$


This yields that $\operatorname{div} \sigma \leq 0$ in the distributional sense, i.e. (2.11). By (4.3), we have

$$
\int_{\Omega}\left\langle\sigma_{k}, D z-D u_{k}\right\rangle d x \geq 0 \text { for all } z \in \mathbb{K}_{\psi}^{F}(\Omega),
$$

since $\mathbb{K}_{\psi}^{F}(\Omega) \subset \mathcal{K}_{\psi}(\Omega)$. Before going on, we note that

$$
\int_{\Omega}\langle\sigma, D u\rangle d x \leq \liminf _{k \rightarrow+\infty} \int_{\Omega}\left\langle\sigma_{k}, D u_{k}\right\rangle d x
$$

Indeed, by (4.9) we get

$$
\left\langle\sigma_{k}, D u_{k}\right\rangle=F_{k}^{*}\left(\sigma_{k}\right)+F_{k}\left(D u_{k}\right) \geq C(L)\left|\sigma_{k}\right|^{q^{\prime}}+L_{0}\left|D u_{k}\right|^{p}-L-\mu_{k} \geq-L-\mu_{k},
$$

where we used $\left(\mathrm{II}_{k}\right)$ and that $F_{k}^{*}(\xi) \geq F^{*}(\xi) \geq C(L)|\xi|^{q^{\prime}}-L$.

Therefore for the sequence of functions $\left\langle\sigma_{k}, D u_{k}\right\rangle$ that converges a.e. to $\langle\sigma, D u\rangle$, we are legitimate to apply Fatou's Lemma to deduce that

$$
\int_{\Omega}\langle\sigma, D u\rangle d x \leq \liminf _{k \rightarrow+\infty} \int_{\Omega}\left(\left\langle\sigma_{k}, D u_{k}\right\rangle+\mu_{k}\right) d x=\liminf _{k \rightarrow+\infty} \int_{\Omega}\left\langle\sigma_{k}, D u_{k}\right\rangle d x,
$$

i.e. (4.17). Since $u_{0} \in W^{1, q}(\Omega)$ and by assumptions (2.3) and (2.4), by Lemma 3.8 there exists a nonincreasing sequence $\psi_{j} \in u_{0}+W_{0}^{1, q}(\Omega)$ such that $\psi_{j} \rightarrow \psi$ a.e. Using (4.3) with $\psi_{j}$ in place of $\varphi$, we get

$$
\int_{\Omega}\left\langle\sigma_{k}, D u_{k}\right\rangle d x \leq \int_{\Omega}\left\langle\sigma_{k}, D \psi_{j}\right\rangle d x
$$

and the weak convergence of $\sigma_{k}$ to $\sigma$ in $L^{q^{\prime}}(\Omega)$ implies that

$$
\liminf _{k} \int_{\Omega}\left\langle\sigma_{k}, D u_{k}\right\rangle d x \leq \liminf _{k} \int_{\Omega}\left\langle\sigma_{k}, D \psi_{j}\right\rangle d x=\int_{\Omega}\left\langle\sigma, D \psi_{j}\right\rangle d x .
$$

Combining (4.10), (4.19) and (4.20) we obtain

$$
\int_{\Omega} F(D u) d x+\int_{\Omega} F^{*}(\sigma) d x=\int_{\Omega}\langle\sigma, D u\rangle d x \leq \int_{\Omega}\left\langle\sigma, D \psi_{j}\right\rangle d x=\llbracket \sigma, \psi_{j} \rrbracket_{u_{0}}(\bar{\Omega})
$$

At this point, passing to the limit as $j \rightarrow \infty$, the monotone convergence theorem yields

$$
\int_{\Omega} F(D u) d x+\int_{\Omega} F^{*}(\sigma) d x \leq \llbracket \sigma, \psi \rrbracket_{u_{0}}(\bar{\Omega})
$$

On the other hand, we have

$$
\begin{aligned}
\llbracket \sigma, \psi \rrbracket_{u_{0}}(\bar{\Omega}) & =\int_{\Omega}\left(\psi-u_{0}^{*}\right) d(-\operatorname{div} \sigma)+\int_{\Omega}\left\langle\sigma, D u_{0}\right\rangle d x \\
& =\int_{\Omega}\left(\psi-u^{*}+u^{*}-u_{0}^{*}\right) d(-\operatorname{div} \sigma)+\int_{\Omega}\left\langle\sigma, D u_{0}\right\rangle d x \\
& \leq \int_{\Omega}\left(u^{*}-u_{0}^{*}\right) d(-\operatorname{div} \sigma)+\int_{\Omega}\left\langle\sigma, D u_{0}\right\rangle d x
\end{aligned}
$$

where we used (3.9) with $u$ in place of $v$. For a standard sequence of mollifiers $\varphi_{\varepsilon}$, let $u_{\varepsilon}=u \star \varphi_{\varepsilon}$, by Lemma 3.5, Fatou's Lemma and next integrating by parts, we have that

$$
\begin{aligned}
\llbracket \sigma, \psi \rrbracket_{u_{0}}(\bar{\Omega}) & \leq \liminf _{\varepsilon} \int_{\Omega}\left(u_{\varepsilon}^{*}-u_{0}^{*}\right) d(-\operatorname{div} \sigma)+\int_{\Omega}\left\langle\sigma, D u_{0}\right\rangle d x \\
& =\liminf _{\varepsilon} \int_{\Omega}\left\langle\sigma, D u_{\varepsilon}\right\rangle d x \leq \liminf _{\varepsilon} \int_{\Omega} F\left(D u_{\varepsilon}\right) d x+\int_{\Omega} F^{*}(\sigma) d x .
\end{aligned}
$$


where we used (3.2). Jensen's inequality implies

$$
\int_{\Omega} F\left(D u_{\varepsilon}\right) d x=\int_{\Omega} F\left(D u \star \varphi_{\varepsilon}\right) d x \leq \int_{\Omega} \varphi_{\varepsilon} \star F(D u) d x
$$

and so

$$
\liminf _{\varepsilon} \int_{\Omega} F\left(D u_{\varepsilon}\right) d x \leq \int_{\Omega} F(D u) d x
$$

Inserting previous estimate in (4.24), we get

$$
\llbracket \sigma, \psi \rrbracket_{u_{0}}(\bar{\Omega}) \leq \int_{\Omega} F(D u) d x+\int_{\Omega} F^{*}(\sigma) d x .
$$

Combining (4.22) with (4.25), we obtain

$$
\llbracket \sigma, \psi \rrbracket_{u_{0}}(\bar{\Omega})=\int_{\Omega} F(D u) d x+\int_{\Omega} F^{*}(\sigma) d x
$$

i.e. the conclusion.

\section{Proof of Theorem 2.2}

This section is devoted to the proof of the regularity result stated in Theorem 2.2.

Proof of Theorem 2.2. We start by observing that every $z \in \mathcal{K}_{\psi}(\Omega)$ belongs to $W^{1, p}(\Omega)$. For every $x_{0} \in \Omega$, we can find a ball $B=B\left(x_{0}, R\right) \subset \Omega$ such that $z_{\left.\right|_{\partial B}} \in W^{1, p}(\partial B)$, see for instance [24]. Then, by Theorem 3.9, $z_{\left.\right|_{\partial B}}$ has an harmonic extension $H$ to $B$, such that $H \in W^{1, \frac{n p}{n-1}}(B)$. Therefore, by the assumption $q<\frac{n p}{n-1}$ and by virtue of $(\mathrm{H} 1)$, we get $F(D H) \in L^{1}(B)$. Using the arguments of the proof of Theorem 2.1, replacing $\Omega$ with $B$ and $u_{0}$ with $H$, give that

$$
F^{*}\left(F^{\prime}(D u)\right) \in L_{\mathrm{loc}}^{1}(\Omega), \quad\left\langle F^{\prime}(D u), D u\right\rangle \in L_{\mathrm{loc}}^{1}(\Omega)
$$

and

$$
\operatorname{div} F^{\prime}(D u) \leq 0
$$

locally, in the distributional sense.

Our next purpose is to prove that $u \in W_{\text {loc }}^{1, q}(\Omega)$. To this aim, let $F_{k}$, $u_{k}$ be respectively the sequence of functionals and their minimizers introduced in the proof of previous Theorem. Let us consider $\varphi_{k}:=u_{k}+t v_{k}$ for a suitable $v_{k} \in W_{0}^{1, p}(\Omega)$ such that

$$
u_{k}-\psi+t v_{k} \geq 0 \quad \text { for } t \in[0,1) .
$$

Such function $\varphi_{k}$ belongs to the admissible class $\mathcal{K}_{\psi}(\Omega)$, because $\varphi_{k}=u_{k}+t v_{k} \geq \psi$ and $\varphi_{k} \in u_{0}+W_{0}^{1, p}(\Omega)$.

Now we fix balls $B_{\frac{R}{2}} \subset B_{\rho} \subset B_{\frac{3}{4} R}$ such that $B_{2 R} \Subset \Omega$ and a cut off function $\eta \in C_{0}^{\infty}\left(B_{\frac{3}{4} R}\right), 0 \leq \eta \leq 1, \eta \equiv 1$ on $B_{\rho}$ such that $|D \eta| \leq \frac{c}{R-\rho}$. Due to the local nature of our results, we suppose $R \leq 1$ without loss of generality. Then, for $|h|<\frac{R}{4}$, we take

$$
v_{k}^{1}(x)=\eta^{2}(x)\left[\left(u_{k}-\psi\right)(x+h)-\left(u_{k}-\psi\right)(x)\right] .
$$

From the regularity of $u_{k}$ and $\psi$, we deduce that $v_{k}^{1} \in W_{0}^{1, p}(\Omega)$. Moreover $v_{k}^{1}$ fulfills (5.1). Indeed, for a.e. $x \in \Omega$ and for any $t \in[0,1)$

$$
\begin{aligned}
u_{k}(x)-\psi(x)+t v_{k}^{1}(x) & =u_{k}(x)-\psi(x)+t \eta^{2}(x)\left[\left(u_{k}-\psi\right)(x+h)-\left(u_{k}-\psi\right)(x)\right] \\
& =t \eta^{2}(x)\left(u_{k}-\psi\right)(x+h)+\left(1-t \eta^{2}(x)\right)\left(u_{k}-\psi\right)(x) \geq 0,
\end{aligned}
$$


because $u_{k} \in \mathcal{K}_{\psi}(\Omega)$. With this choice in (4.3), we obtain

$$
0 \leq \int_{\Omega}\left\langle F_{k}^{\prime}(D u(x)), D\left[\eta^{2}(x)\left[\left(u_{k}-\psi\right)(x+h)-\left(u_{k}-\psi\right)(x)\right]\right]\right\rangle d x .
$$

On the other hand, if we introduce

$$
v_{k}^{2}(x)=\eta^{2}(x-h)\left[\left(u_{k}-\psi\right)(x-h)-\left(u_{k}-\psi\right)(x)\right],
$$

then $v_{k}^{2} \in W_{0}^{1, p}(\Omega)$ and it satisfies condition (5.1), as long as $v_{k}^{2}(x)=v_{k}^{1}(x-h)$. Choosing in (4.3) as test function $\varphi_{k}=u_{k}+t v_{k}^{2}$, where $v_{k}^{2}$ is defined in (5.4), we get

$$
0 \leq \int_{\Omega}\left\langle F_{k}^{\prime}\left(D u_{k}(x)\right), D\left[\eta^{2}(x-h)\left[\left(u_{k}-\psi\right)(x-h)-\left(u_{k}-\psi\right)(x)\right]\right]\right\rangle d x,
$$

Changing variable we get

$$
0 \leq \int_{\Omega}\left\langle F_{k}^{\prime}\left(D u_{k}(x+h)\right), D\left[\eta^{2}(x)\left[\left(u_{k}-\psi\right)(x)-\left(u_{k}-\psi\right)(x+h)\right]\right]\right\rangle d x .
$$

Thus by adding (5.3) and (5.5), we obtain

$$
\begin{aligned}
0 \leq & \int_{\Omega}\left\langle F_{k}^{\prime}\left(D u_{k}(x)\right), D\left[\eta^{2}(x)\left[\left(u_{k}-\psi\right)(x+h)-\left(u_{k}-\psi\right)(x)\right]\right]\right\rangle d x \\
& +\int_{\Omega}\left\langle F_{k}^{\prime}\left(D u_{k}(x+h)\right), D\left[\eta^{2}(x)\left[\left(u_{k}-\psi\right)(x)-\left(u_{k}-\psi\right)(x+h)\right]\right]\right\rangle d x \\
= & \int_{\Omega}\left\langle F_{k}^{\prime}(D u(x))-F_{k}^{\prime}\left(D u_{k}(x+h)\right), D\left[\eta^{2}(x)\left[\left(u_{k}-\psi\right)(x+h)-\left(u_{k}-\psi\right)(x)\right]\right]\right\rangle d x,
\end{aligned}
$$

which implies

$$
\begin{aligned}
0 \geq & \int_{\Omega}\left\langle F_{k}^{\prime}\left(D u_{k}(x+h)\right)-F_{k}^{\prime}\left(D u_{k}(x)\right), \eta^{2}(x) D\left[\left(u_{k}-\psi\right)(x+h)-\left(u_{k}-\psi\right)(x)\right]\right\rangle d x \\
& +\int_{\Omega}\left\langle F_{k}^{\prime}\left(D u_{k}(x+h)\right)-F_{k}^{\prime}\left(D u_{k}(x)\right), 2 \eta(x) D \eta(x)\right. \\
& \left.\cdot\left[\left(u_{k}-\psi\right)(x+h)-\left(u_{k}-\psi\right)(x)\right]\right\rangle d x .
\end{aligned}
$$

The previous inequality can be rewritten as follows

$$
\begin{aligned}
0 \geq & \int_{\Omega}\left\langle F_{k}^{\prime}\left(D u_{k}(x+h)\right)-F_{k}^{\prime}\left(D u_{k}(x)\right), \eta^{2}\left(D u_{k}(x+h)-D u_{k}(x)\right)\right\rangle d x \\
& -\int_{\Omega}\left\langle F_{k}^{\prime}\left(D u_{k}(x+h)\right)-F_{k}^{\prime}\left(D u_{k}(x)\right), \eta^{2}(D \psi(x+h)-D \psi(x))\right\rangle d x \\
& +\int_{\Omega}\left\langle F_{k}^{\prime}\left(D u_{k}(x+h)\right)-F^{\prime}\left(D u_{k}(x)\right), 2 \eta D \eta \tau_{h}\left(u_{k}-\psi\right)\right\rangle d x \\
= & : I+I I+I I I,
\end{aligned}
$$

that yields

$$
I \leq|I I|+|I I I|
$$

The ellipticity of $F_{k}$ expressed by (II) of Lemma 3.7 and Lemma 3.1 imply

$$
I \geq c(p, \nu) \int_{\Omega} \eta^{2}\left|\tau_{h} V_{p}\left(D u_{k}\right)\right|^{2} d x .
$$

For the estimation of $I I$ and $I I I$, we use Hölder's inequality to deduce that

$$
|I I|+|I I I| \leq\left(\int_{\Omega} \eta^{q^{\prime}}\left|F_{k}^{\prime}\left(D u_{k}(x)\right)\right|^{q^{\prime}} d x\right)^{\frac{1}{q^{\prime}}}\left(\int_{\Omega} \eta^{q}\left|\tau_{h} D \psi\right|^{q} d x\right)^{\frac{1}{q}}
$$




$$
\begin{aligned}
& +\left(\int_{\Omega} \eta^{q^{\prime}}\left|F_{k}^{\prime}\left(D u_{k}(x)\right)\right|^{q^{\prime}} d x\right)^{\frac{1}{q^{\prime}}}\left(\int_{\Omega}|D \eta|^{q}\left|\tau_{h} u_{k}\right|^{q} d x\right)^{\frac{1}{q}} \\
& +\left(\int_{\Omega} \eta^{q^{\prime}}\left|F_{k}^{\prime}\left(D u_{k}(x)\right)\right|^{q^{\prime}} d x\right)^{\frac{1}{q^{\prime}}}\left(\int_{\Omega}|D \eta|^{q}\left|\tau_{h} \psi\right|^{q} d x\right)^{\frac{1}{q}} \\
\leq & \left(\int_{B_{R}}\left|F_{k}^{\prime}\left(D u_{k}(x)\right)\right|^{q^{\prime}} d x\right)^{\frac{1}{q^{\prime}}} \\
& \cdot\left[\left(\int_{B_{R}}\left|\tau_{h} D \psi\right|^{q} d x\right)^{\frac{1}{q}}+\frac{c}{R-\rho}\left(\int_{B_{R}}\left|\tau_{h} u_{k}\right|^{q} d x\right)^{\frac{1}{q}}+\frac{c}{R-\rho}\left(\int_{B_{R}}\left|\tau_{h} \psi\right|^{q} d x\right)^{\frac{1}{q}}\right],
\end{aligned}
$$

where we used the properties of $\eta$ and that, since $u \in W^{1, p}(\Omega)$ and $q<\frac{p n}{n-1}<\frac{p n}{n-p}$ we have that $\tau_{h} u \in L^{q}(\Omega)$. We used also that

$$
\int_{B_{\frac{3}{4} R}}|f(x+h)|^{q^{\prime}} d x \leq c \int_{B_{R}}|f(x)|^{q^{\prime}} d x
$$

for $|h|<\frac{R}{4}$. Denoted by $H_{u}$ the harmonic extension of $u$ to the ball $B_{2 R}$, we can use (4.12) with $H_{u}$ in place of $u_{0}$ to obtain

$$
\left(\int_{B_{R}}\left|F_{k}^{\prime}\left(D u_{k}(x)\right)\right|^{q^{\prime}} d x\right)^{\frac{1}{q^{\prime}}} \leq C\left(\int_{B_{2 R}} F\left(D H_{u}(x)\right) d x\right)^{\frac{1}{q^{\prime}}}
$$

and so

$$
\begin{aligned}
|I I|+|I I I| & \left(\int_{B_{2 R}} F\left(D H_{u}(x)\right) d x\right)^{\frac{1}{q^{\prime}}} \\
\cdot & {\left[\left(\int_{B_{R}}\left|\tau_{h} D \psi\right|^{q} d x\right)^{\frac{1}{q}}+\frac{c}{R-\rho}\left(\int_{B_{R}}\left|\tau_{h} u_{k}\right|^{q} d x\right)^{\frac{1}{q}}+\frac{c}{R-\rho}\left(\int_{B_{R}}\left|\tau_{h} \psi\right|^{q} d x\right)^{\frac{1}{q}}\right] } \\
\leq & C\left(\int_{B_{2 R}}\left(\left|D H_{u}(x)\right|^{q}+1\right) d x\right)^{\frac{1}{q^{\prime}}} \\
\cdot & {\left[\left(\int_{B_{R}}\left|\tau_{h} D \psi\right|^{q} d x\right)^{\frac{1}{q}}+\frac{c}{R-\rho}\left(\int_{B_{R}}\left|\tau_{h} u_{k}\right|^{q} d x\right)^{\frac{1}{q}}+\frac{c}{R-\rho}\left(\int_{B_{R}}\left|\tau_{h} \psi\right|^{q} d x\right)^{\frac{1}{q}}\right] }
\end{aligned}
$$

where we used the right inequality in (H1). Therefore, plugging (5.8) and (5.10) in (5.7) and using that $\eta \equiv 1$ on $B_{\rho}$, we obtain

$$
\begin{aligned}
& c(p, \nu) \int_{B_{\rho}}\left|\tau_{h} V_{p}\left(D u_{k}\right)\right|^{2} \leq C\left(\int_{B_{2 R}}\left(\left|D H_{u}(x)\right|^{q}+1\right) d x\right)^{\frac{1}{q^{\prime}}} \\
& \cdot\left[\left(\int_{B_{R}}\left|\tau_{h} D \psi\right|^{q} d x\right)^{\frac{1}{q}}+\frac{c}{R-\rho}\left(\int_{B_{R}}\left|\tau_{h} u_{k}\right|^{q} d x\right)^{\frac{1}{q}}+\frac{c}{R-\rho}\left(\int_{B_{R}}\left|\tau_{h} \psi\right|^{q} d x\right)^{\frac{1}{q}}\right] .
\end{aligned}
$$

Using the assumption on $\psi$, i.e. $D \psi \in W_{\text {loc }}^{1, q}(\Omega)$, and the embedding of Theorem 3.6 with

$$
\alpha=1-n\left(\frac{1}{p}-\frac{1}{q}\right)>0 \quad \text { and } \quad q=\frac{n p}{n-\alpha p}
$$


we arrive at

$$
\begin{aligned}
& \int_{B_{\rho}}\left|\tau_{h} V_{p}\left(D u_{k}\right)\right|^{2} \leq C\left(\int_{B_{2 R}}\left(\left|D H_{u}(x)\right|^{q}+1\right) d x\right)^{\frac{1}{q^{\prime}}} \\
& \cdot\left[|h|\left(\int_{B_{2 R}}\left|D^{2} \psi\right|^{q} d x\right)^{\frac{1}{q}}+\frac{C|h|^{\alpha}}{R-\rho}\left(\int_{B_{R}}|D u|^{p} d x\right)^{\frac{1}{p}}+\frac{C|h|}{R-\rho}\left(\int_{B_{2 R}}|D \psi|^{q} d x\right)^{\frac{1}{q}}\right] \\
& \leq C|h|^{\alpha}\left(\int_{B_{2 R}}\left(\left|D H_{u}(x)\right|^{q}+1\right) d x\right)^{\frac{1}{q^{\prime}}}\left(\|D u\|_{L^{p}\left(B_{2 R}\right)}+\|\psi\|_{W^{2, q}\left(B_{2 R}\right)}\right) .
\end{aligned}
$$

Estimate (5.12) implies that $V_{p}\left(D u_{k}\right) \in B_{2, \infty, \text { loc }}^{\frac{\alpha}{2}}(\Omega)$ and therefore, again by Theorem 3.6, we have

$$
V_{p}\left(D u_{k}\right) \in L_{\text {loc }}^{s}(\Omega) \quad \text { for every } s<\frac{2 n}{n-\alpha}
$$

and so by the definition of the function $V_{p}(\xi)$ and recalling the value of $\alpha$

$$
D u_{k} \in L_{\text {loc }}^{t}(\Omega) \text { for every } t<\frac{n p}{n\left(1+\frac{1}{p}-\frac{1}{q}\right)-1} .
$$

Following [2], we now define the sequence of exponents

$$
p=p_{0}, \quad p_{j}=\frac{n p}{n\left(1+\frac{1}{p_{j-1}}-\frac{1}{q}\right)-1}=\frac{n p}{n-1-\frac{n}{q}+\frac{n}{p_{j-1}}} .
$$

Arguing inductively, we have that

$$
D u_{k} \in L_{\text {loc }}^{t}(\Omega) \text { for every } t<p_{j-1} \Longrightarrow D u_{k} \in L_{\text {loc }}^{t}(\Omega) \text { for every } t<p_{j} .
$$

Note that, in case $1<p<q \leq \frac{n}{n-1}$ (that obviously implies $n-1-\frac{n}{q} \leq 0$ ), we have

$$
p_{j} \geq \frac{n p}{\frac{n}{p_{j-1}}}=p \cdot p_{j-1} \Longrightarrow p_{j} \geq p_{0} \cdot p^{j-1}=p^{j}
$$

and so $p_{j}$ is an increasing sequence such that

$$
p_{j} \rightarrow \infty \text {. }
$$

Therefore, there exists $\bar{j}$ such that to have $q<p^{j}$, for every $j>\bar{j}$.

In case $\frac{n}{n-1}<q<\frac{p n}{n-1}$, one can easily check that if $p_{j-1}<\frac{n(p-1)}{n-1-\frac{n}{q}}$, then

$$
p_{j} \nearrow \frac{n(p-1)}{n-1-\frac{n}{q}}
$$

and that, for $q<\frac{p n}{n-1}$, we have

$$
q<\frac{n(p-1)}{n-1-\frac{n}{q}}
$$

Hence, also in this case there exists $\bar{j}$ such that to have $q<p_{j}$, for every $j>\bar{j}$.

Therefore, by virtue of (5.13), iterating estimate (5.12) we deduce that the sequence $u_{k}$ is bounded in $W^{1, q}\left(B_{R}\right)$ and therefore its limit $u$ also belongs to $W^{1, q}\left(B_{R}\right)$. This conclude the proof.

Acknowledgements. The work of the authors is supported by GNAMPA (Gruppo Nazionale per l'Analisi Matematica, la Probabilità e le loro Applicazioni) of INdAM (Istituto Nazionale di Alta Matematica), by MIUR through the project FFABR and by the University of Modena and Reggio Emilia through the project FAR2019 
"Equazioni differenziali: problemi evolutivi, variazionali ed applicazioni" (coord. Prof. M. Manfredini) by the University of Naples "Federico II" through the project 000022-ALTRI_CDA_75_2021_FRA_PASSARELLI (coord. A. Passarelli di Napoli). This research was performed while A. Passarelli di Napoli was visiting the University of Modena and Reggio Emilia and M. Eleuteri was visiting the University of Naples "Federico II". The hospitality of both Institutions is warmly adknowledged.

\section{References}

[1] Anzellotti, G.: Pairing between measures and bounded functions and compensated compactness. - Ann. Mat. Pura Appl. 135:4, 1984, 293-318.

[2] Carozza, M., J. Kristensen, and A. Passarelli di Napoli: Regularity of minimizers of autonomous convex variational integrals. - Ann. Sc. Norm. Super. Pisa Cl. Sci. (5) 13:4, 2014, 1065-1089.

[3] Carozza, M., J. Kristensen, and A. Passarelli di Napoli: On the validity of the Euler Lagrange system. - Comm. Pure Appl. Anal. 14:1, 2018, 51-62.

[4] Carozza, M., J. Kristensen, and A. Passarelli di Napoli: A trace preserving operator and applications. - J. Math. Anal. Appl. 501, 2021, 124170.

[5] Caselli, M., M. Eleuteri, and A. Passarelli Di Napoli: Regularity results for a class of obstacle problems with $p, q$-growth conditions. - ESAIM Control Optim. Calc. Var. 27, 2021, 27.

[6] Chen, G.-Q., and H. FRID: Divergence-measure fields and hyperbolic conservation laws. Arch. Ration. Mech. Anal. 147:2, 1999, 89-118.

[7] Cianchi, A.: A fully anisotropic Sobolev inequality. - Pacific J. Math. 196:2, 2000, 283-294.

[8] Dacorogna, B.: Direct methods in the calculus of variations. - Springer, 1989.

[9] Dal Maso, G.: On the integral representation of certain local functionals. - Ric. Mat. 32:1, 1983, 85-113.

[10] Ekeland, I., and R. Temam: Convex analysis and variational problems. - Classics Appl. Math. 28, SIAM, Philadelphia, 1999.

[11] Evans, L. C., and R. F. Gariepy: Measure theory and fine properties of functions. - CRC Press, Taylor \& Francis Group, 2015.

[12] Giusti, E.: Direct methods in the calculus of variations. - World Sci. Publ. Co., 2003.

[13] Haroske, D.: Envelopes and sharp embeddings of function spaces. - Chapman and Hall CRC, 2006.

[14] Heinonen, J., T. KilpeläInen, and O. Martio: Nonlinear potential theory of degenerate elliptic equations. - Dover Publications, 1993.

[15] Kohn, R. V., and R. Temam: Dual spaces of stresses and strains, with applications to Hencky plasticity. - Appl. Math. Optim. 10:1, 1983, 1-35.

[16] Kristensen, J., and G. Mingione: Boundary regularity in variational problems. - Arch. Ration. Mech. Anal. 198, 2010, 369-455.

[17] Marcellini, P.: Regularity of minimizers of integrals of the calculus of variations with nonstandard growth conditions. - Arch. Ration. Mech. Anal. 105:3, 1989, 267-284.

[18] Marcellini, P.: Regularity and existence of solutions of elliptic equations with $p, q$-growth conditions. - J. Differential Equations 90:1, 1991, 1-30.

[19] Mercaldo, A., S. Segura de León, and C. Trombetti: On the solutions to 1-Laplace equation with $L^{1}$ data. - J. Funct. Anal. 256:8, 2009, 2387-2416.

[20] Rockafeller, R. T.: Convex analysis. - Princeton Univ. Press, 1997.

[21] Scheven, C., and T. Schmidt: BV supersolutions to equation of 1-Laplace and minimal surface type. - J. Differential Equations 261, 2016, 1904-1932. 
[22] Scheven, C., and T. Schmidt: On the dual formulation of obstacle problems for the total variation and the area functional. - Ann. Inst. H. Poincaré Anal. Non Linéaire 35, 2018, 11751207.

[23] Tartar, L.: An introduction to Sobolev spaces and interpolations spaces. - Lecture Notes of the Unione Matematica Italiana 3, Springer, 2007.

[24] Ziemer, W. P.: Weakly differentiable functions. - Grad. Texts in Math. 120, Springer-Verlag, 1989.

Received 29 September 2020 • Revised received 18 June 2021 • Accepted 6 July 2021

Published online 12 February 2022

Michela Eleuteri

Università degli Studi di Modena E Reggio Emilia

Dipartimento di Scienze Fisiche, Informatiche e Matematiche

Via Campi 213/b, 41125 Modena, Italy

michela.eleuteri@unimore.it

Antonia Passarelli di Napoli

Università degli Studi di Napoli "Federico II"

Dipartimento di Matematica e Applicazioni "R. Caccioppoli"

Via Cintia, 80126, Napoli, Italy

antonia.passarellidinapoli@unina.it 